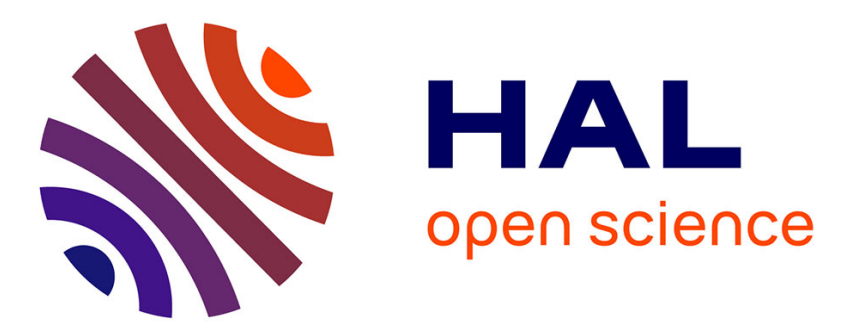

\title{
Crystal Stability of Diblock Copolymer Micelles in Solution
}

John Jairo Molina, Carlo Pierleoni, Barbara Capone, Jean-Pierre Hansen, Igor Oliveira

\section{- To cite this version: \\ John Jairo Molina, Carlo Pierleoni, Barbara Capone, Jean-Pierre Hansen, Igor Oliveira. Crystal Stability of Diblock Copolymer Micelles in Solution. Molecular Physics, 2009, 107 (04-06), pp.535- 548. 10.1080/00268970902877779 . hal-00513281}

\section{HAL Id: hal-00513281 \\ https://hal.science/hal-00513281}

Submitted on 1 Sep 2010

HAL is a multi-disciplinary open access archive for the deposit and dissemination of scientific research documents, whether they are published or not. The documents may come from teaching and research institutions in France or abroad, or from public or private research centers.
L'archive ouverte pluridisciplinaire HAL, est destinée au dépôt et à la diffusion de documents scientifiques de niveau recherche, publiés ou non, émanant des établissements d'enseignement et de recherche français ou étrangers, des laboratoires publics ou privés. 


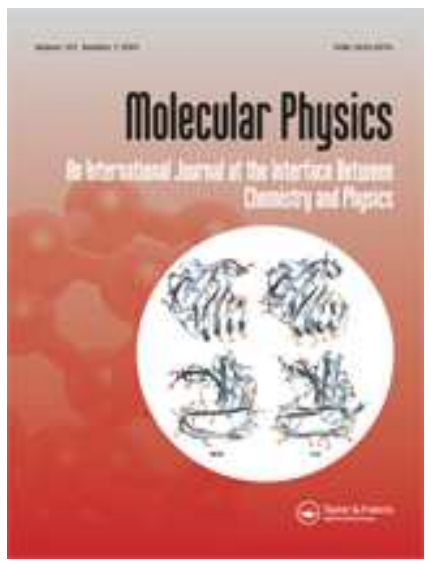

Crystal Stability of Diblock Copolymer Micelles in Solution

\begin{tabular}{|r|l|}
\hline Journal: & Molecular Physics \\
\hline Manuscript ID: & TMPH-2009-0035.R1 \\
\hline Manuscript Type: & Special Issue Paper - Dr. Jean-Jacques Weis \\
\hline Author: & 05-Feb-2009 \\
\hline Complete List of Authors: & $\begin{array}{l}\text { Molina, John; Université Pierre et Marie Curie, Laboratoire Liquides } \\
\text { Ioniques et Interfaces Chargées; University of Rome "La Sapienza", } \\
\text { Physics Dept. } \\
\text { Pierleoni, Carlo; University of L'Aquila, CNISM and Physics Dept. } \\
\text { Capone, Barbara; Cambridge, University Chemical Laboratory } \\
\text { Hansen, Jean-Pierre; Cambridge, University Chemical Laboratory; } \\
\text { Université Pierre et Marie Curie, Laboratoire Liquides Ioniques et } \\
\text { Interfaces Chargées } \\
\text { Oliveira, Igor; University of Twente, Computational Biophysics; } \\
\text { University of Rome "La Sapienza", Physics Dept. }\end{array}$ \\
\hline $\begin{array}{l}\text { TMPH-2009-0035.R1.tar.gz } \\
\text { Kotey }\end{array}$ & \begin{tabular}{l} 
diblock copolymers, micelles, self-aggregation, effective potentials \\
\hline to PDF. You must view these files (e.g. movies) online.
\end{tabular} \\
\hline \hline
\end{tabular}

\section{(5) ScholaroNE \\ Manuscript Central}




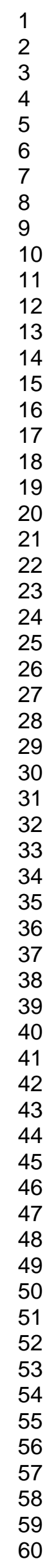

URL: http://mc.manuscriptcentral.com/tandf/tmph 


\title{
FULL ARTICLE
}

\section{Crystal Stability of Diblock Copolymer Micelles in Solution}

\author{
John Jairo Molina ${ }^{\dagger \ddagger *}$, Carlo Pierleoni ${ }^{\S}$, Barbara Capone ${ }^{\uparrow}$, Jean-Pierre Hansen ${ }^{\uparrow \dagger}$, and \\ Igor Saulo Santos de Oliveirall \\ $\dagger$ Laboratoire Liquides Ioniques et Interfaces Chargées, Université P. et M. Curie, 4 place \\ Jussieu, F-75252 Paris CEDEX 05, France \\ $\ddagger$ Physics Dept., University of Rome "La Sapienza", I-00185 Rome, Italy \\ $\S$ CNISM and Physics Dept. University of L'Aquila, I-67010 L'Aquila, Italy \\ ฯ University Chemical Laboratory, Lensfield Road, Cambridge CB2 1EW, United \\ Kingdom \\ \| Computational Biophysics, University of Twente, P.O. Box 217, 7500 AE, Enschede, \\ The Netherlands \\ (Received 00 Month 200x; final version received 00 Month 200x)
}

\begin{abstract}
We investigate the relative stability of the disordered phase and of four crystal structures of micelles resulting from the self-assembly of $A B$ diblock copolymers in semi-dilute solutions. Starting from the micelle-micelle pair distribution functions determined previously in the disordered fluid phase by Monte Carlo simulations of a coarse-grained model of diblock copolymers, we extract effective pair potentials $v(r)$ between micelle centres of mass by a novel extrapolation/inversion technique. These $v(r)$ are used in extensive Monte Carlo simulations of micellar assemblies to determine the structures, mean-square displacements, and free energies of four ordered phases including FCC, BCC, diamond and the less common A15 crystals. For micelle densities close to melting, we predict the most stable structures to be FCC and A15, with the latter phase having the lowest free energy for micelles with small cores and large coronae, in agreement with recent predictions for micelles forming in copolymer melts (G.M. Grason et al., Phys. Rev. Lett. 91, 058304 (2003))
\end{abstract}

Keywords: diblock copolymers; micelles; self-aggregation; effective potentials

\section{Introduction}

Diblock copolymers are known to self-assemble into ordered or disordered supramolecular aggregates or microphases, including micelles, lamellae, cylinders or bicontinuous structures, both in the melt $[1,2]$ and in selective solvents [3, 4]. Selfconsistent mean-field theory, which is well adapted to melts [5], does not apply to semi-dilute solutions of copolymers, where concentration fluctuations are dominant, as signalled by a large osmotic compressibility. Molecular simulation is well adapted to copolymer solutions, but due to the large number of copolymers required to observe micro-phase separation, simulations of fully atomistic, monomer-level models are restricted to rather short copolymer chains [6-8].

To overcome this limitation and model long chains, we have developed a systematic coarse-graining strategy which reduces the computational effort by orders of

\footnotetext{
*Corresponding author: john.molina@etu.upmc.fr
} 
magnitude [9-11]. The basic idea generalises earlier work on homopolymer solutions, where each polymer is represented by a single blob, and interactions between polymer coils reduce to an effective pair potential between the centres of mass (CM) of the coils [12-14].

The effective pair potential $v(r)$ is calculated from a fully microscopic, monomerlevel simulation of two isolated polymer chains (corresponding to the infinite dilution limit); $v(r)$ is easily extracted as the potential of mean force associated with the measured CM-CM pair distribution function $g(r)$, according to:

$$
v(r)=-k_{B} T \ln g(r)
$$

where $k_{B}$ is the Boltzmann's constant and $T$ the temperature. Even for polymers with excluded volume correlations between monomers, $v(r)$ turns out to be soft and finite at full overlap (i.e. for $r=0$ ) [13-15]. In fact $v(r)$ turns out to be accurately represented by a gaussian function of amplitude $v(r=0) \simeq 2 k_{B} T$ and width practically equal to the polymer radius of gyration $R_{g}$. At higher polymer concentration $v(r)$ is extracted from $g(r)$ by the hypernetted chain (HNC) integral equation [16] and turns out to depend weakly on concentration [14]. This concentration dependence of the effective interaction may be eliminated by switching to a multi-blob representation of long polymer chains [17].

Returning to copolymers, the minimal coarse-grained model of an $A B$ diblock copolymer is a "soft dumbbell" representation, where each of the two blocks is represented by a single effective blob, and the two blobs are tethered by an entropic spring [9-11]. In references [10] and [11], this representation was applied to a simple athermal copolymer model, mimicking $A B$ copolymers in a selective solvent, with ideal (random walk) $A$-blocks (corresponding roughly to $\theta$-solvent conditions) and self-avoiding walk (SAW) $B$-blocks (corresponding to good solvent conditions); $A$ and $B$ blocks are taken to be mutually avoiding, and the corresponding copolymer solution is referred to as the ISS model. The model is characterised by three effective intermolecular blob-blob pair potentials $v_{A A}(r), v_{A B}(r)$, and $v_{B B}(r)$, and by an intramolecular tethering potential $\phi_{A B}(r)$, which are determined from the low density intermolecular pair distribution functions $g_{\alpha \beta}(r)$ and the intramolecular distribution function $s_{A B}(r)$ by an exact inversion procedure [9]. The resulting soft-dumbbell representation was explored by extensive Monte Carlo (MC) simulations in refs. [10] and [11] for several values of the size ratio:

$$
f=\frac{L_{A}}{L_{A}+L_{B}}
$$

where $L_{A}$ and $L_{B}$ are the lengths of the $A$ and $B$ strands. It was shown that at an $f$-dependent critical micellar concentration $(\mathrm{cmc})$, the copolymers self-assembled into polydisperse spherical micelles with $A$-cores and $B$-coronae, and typical aggregation numbers $n \simeq 100$. The micelles first arranged into a disordered, fluid-like structure, but upon further increasing the copolymer concentration, the micelles are found to undergo a disorder-order transition to a crystalline structure. The symmetry of the crystal which is observed is strongly dependent on the number of self-assembled micelles which form in the simulation box and the compatibility of the structure with the imposed periodic boundary conditions [10, 11]. The crystals which form may hence be merely metastable, and it would clearly be of interest to determine the most stable (lowest free energy) structure at a given micelle density as determined by the copolymer concentration and the mean aggregation number $n$. This can be achieved by going one step further in the coarse-graining strategy, i.e. by determining the effective pair interaction between micelle CM's, 
and then by carrying out MC simulations of large arrays of micelles to determine the relative stability of standard crystal structures, including FCC, BCC, and diamond structures, as well as the less familiar A15 structure [18, 19], which has been shown to be the most stable micellar crystal in the melt by a geometric argument and a self-consistent mean-field calculation [19-21]. The same phase has been shown to be stable for star polymer solutions at very high densities, at least at zero temperature [22]. Since these arguments do not, a priori, apply to diblock copolymer solutions, the main objective of the present paper is to investigate the stability of the above crystal lattices by numerical simulation. The inversion procedure used to extract the effective micelle-micelle pair potential is detailed in Section 2. MC results for the micelle pair distribution functions in the ordered phases and for the Lindemann ratios are presented in Section 3 while free energy calculations are detailed in Section 4. The predictions for the relative stabilities of the four crystal structures and the disordered (fluid) phase are summarised in Section 5 while a discussion and conclusions are presented in Section 6.

\section{Extracting effective pair potentials from structural data}

In references [10] and [11] we reported the results of extensive MC simulations of systems of 5000 "soft dumbbells" representing ISS diblock copolymers over a range of size ratios $0.2 \leq f \leq 0.8$ and of copolymer densities $\rho / \rho *$, where $\rho$ is the number of copolymers per unit volume, while $\rho *=3 / 4 \pi R_{g}^{3}$ is the overlap density, i.e. the density at which neighbouring copolymers start to overlap; $R_{g}$ is the radius of gyration of an isolated diblock copolymer, and will henceforth be our unit of length. Note that for sufficiently long copolymers $\left(L=L_{A}+L_{B} \gg\right.$ monomer size), their size uniquely determines $R_{g}$, so that any copolymer size dependence scales with $R_{g}$. For copolymer concentrations beyond the cmc, when most copolymers have aggregated into micelles, the MC simulations yield the pair distribution function $g(r)$ of micelle CM's. This $g(r)$ must be understood as being averaged over all micelles, irrespective of their size (i.e. their instantaneous aggregation number, which fluctuates) and over all configurations of the remaining copolymers.

According to a uniqueness theorem [23] there is a unique effective pair potential associated with a measured $g(r)$, irrespective of the microscopic Hamiltonian governing the underlying physical system. We are hence faced with an inverse problem, namely how to extract the effective micelle-micelle pair potential from the pair distribution function provided by the MC simulations of self-assembling soft dumbbells representing the diblock copolymers. Several inversion methods, usually based on fluid integral equations, have been implemented in the past with varying degrees of success [24-28]. The most accurate of the inversion schemes involves an iterative predictor-corrector procedure based on MC simulations [26, 27]. All inversion procedures require the input $g(r)$ to be known over a wide range of inter-particle distances, up to values of $r$ where $g(r)$ has converged to its asymptotic value 1 . This is unfortunately not the case for the micelle-micelle $g(r)$ generated in the MC simulations of references $[10,11]$ because of limitations on system size; even with 5000 copolymers, the number of micelles which form is of the order of 50 , and for such a system size, $g(r)$ still differs significantly from 1 at densities close to freezing, even at the largest accessible distance $r=L / 2$, where $L$ is the size of the cubic simulation cell. Hence, before attempting an inversion, the MC data for $g(r)$ must be extended to distances $r>L / 2$. Such an extension is easily achieved, provided the underlying pair potential $v(r)$ is known [29], which is precisely not the case in the present situation.

We have overcome this problem by devising two independent extrapolation 


\section{Molecular Physics}

4

J.J. Molina et al.

schemes. The first scheme is based on the observation that the static structure factor $S(k)$, which is the Fourier transform (FT) of the pair correlation function $h(r)=g(r)-1$, is expected to behave smoothly at small wavenumbers $k \simeq k_{\min }=2 \pi / L$, which is the smallest wavenumber accessible in direct MC estimates of $S(k)$ calculated via [16]:

$$
\begin{aligned}
S(k) & =\frac{1}{N}\left\langle\rho_{\vec{k}} \rho_{-\vec{k}}\right\rangle \\
& =1+\frac{1}{N}\left\langle\sum_{i \neq j} e^{i \vec{k} \cdot\left(\vec{r}_{i}-\vec{r}_{j}\right)}\right\rangle
\end{aligned}
$$

where $N$ is the mean number of micelles in the simulation box. If the (unknown) pair potential is assumed to be of sufficiently short range, then $S(k)$ admits a Taylor expansion in even powers of $k$ at small $k$ :

$$
S(k)=S_{0}+S_{2} k^{2}+S_{4} k^{4}+S_{6} k^{6}+S_{8} k^{8}+\mathcal{O}\left(k^{10}\right)
$$

where $S_{0}=S(k=0)$ is related to the osmotic compressibility of the micelles. If $S_{\text {mod }}(k)$ refers to the above Taylor expansion, and the MC data for $k \geq \widetilde{k}$ are referred to as $S_{d a t}(k)$, then the total structure factor is taken to be:

$$
S(k)=\left\{\begin{array}{l}
S_{\text {mod }}(k), k<\widetilde{k} \\
S_{\text {dat }}(k), k \geq \widetilde{k}
\end{array}\right.
$$

with $\widetilde{k}$ chosen to ensure continuity of $S(k)$. The corresponding pair correlation function is then given by the FT of $(5)^{1}$, i.e.:

$$
\begin{aligned}
\rho h(r)=\frac{4 \pi}{(2 \pi)^{3}}\{ & \int_{0}^{\widetilde{k}} \mathrm{~d} k \frac{k \sin (k r)}{r}\left[S_{\text {mod }}(k)-1\right] \\
& \left.+\int_{\widetilde{k}}^{\infty} \mathrm{d} k \frac{k \sin (k r)}{r}\left[S_{\text {dat }}(k)-1\right]\right\}
\end{aligned}
$$

If $h_{\text {dat }}(r)$ are the MC data for the pair correlation function, available up to $r=L / 2$, we define the two functions:

$$
\begin{gathered}
\Phi_{d a t}(r)=\rho h_{d a t}(r)-\frac{4 \pi}{(2 \pi)^{3}} \int_{\widetilde{k}}^{\infty} \mathrm{d} k \frac{k \sin (k r)}{r}\left[S_{d a t}(k)-1\right] \\
\Phi_{\text {mod }}(r)=\int_{0}^{\widetilde{k}} \mathrm{~d} k \frac{k \sin (k r)}{r}\left[S_{\text {mod }}(k)-1\right]
\end{gathered}
$$

In view of eq. (6), we now require that:

$$
\Phi_{d a t}(r)=\Phi_{\text {mod }}(r)
$$
${ }_{1}^{1}$ in order to perform the Fourier integrals in eq. (6), $S_{d a t}(k)$ was determined on a uniform grid by a cubic
spline interpolation of the coarser grid of MC data. 
This is achieved by minimising the following weighted mean square deviation with respect to the expansion coefficients $S_{2 l}$ in eq. (4):

$$
\begin{aligned}
\chi^{2} & =\sum_{k=k_{\min }}^{k_{\max }} \frac{\left[S_{d a t}(k)-S_{\text {mod }}(k)\right]^{2}}{\delta S_{d a t}^{2}(k)} \\
& +\sum_{r=r_{\min }}^{r_{\max }} \frac{\left[\Phi_{d a t}(r)-\Phi_{\bmod }(r)\right]^{2}}{\delta \Phi_{d a t}^{2}(r)}
\end{aligned}
$$

where $\delta S_{d a t}^{2}(k)$ and $\delta \Phi_{d a t}^{2}(r)$ are statistical errors of the MC data; $k_{\text {min }}=2 \pi / L$, $k_{\max }=\widetilde{k}, r_{\max }=L / 2$ and $r_{\min }$ is the distance at which the MC-generated $g(r)$ differs significantly from zero.

An alternative extrapolation procedure is based on the assumption that the (unknown) $v(r)$ is of finite range, i.e. $v(r)=0, r>r_{c}$. If $r_{c}$ is the node of the MCgenerated correlation function $h_{d a t}(r)$ closest to $L / 2$, then we adopt the following (approximate) closure relation:

$$
\left\{\begin{array}{l}
h(r)=h_{d a t}(r) ; r<r_{c} \\
c(r)=0 \quad ; r>r_{c}
\end{array}\right\}
$$

where $c(r)$ is the direct correlation function related to $h(r)$ by the familiar OrnsteinZernike (OZ) relation [16] (in $k$-space):

$$
\widehat{h}(k)=\widehat{c}(k)+\rho \widehat{c}(k) \widehat{h}(k)
$$

Eqs. (10) and (11) form a closed set, which can be solved by iteration to yield $h(r)$ for $r>r_{c}$ and $c(r)$ for $r<r_{c}$. The two extrapolation procedures yield similar results. Yet another extrapolations scheme, based on fitting the available MC data to an exponentially damped sinusoidal function, was found to be inadequate because the simulations yield too few oscillations in $g(r)$, due to the small system size. Once $h(r)$ and $c(r)$ are known for all $r$, the effective potential between micelles can be extracted from the HNC closure:

$$
v(r)=k_{B} T[h(r)-c(r)-\ln (1+h(r))]
$$

This closure is only approximate, and has proved inadequate in earlier attempts to extract pair potentials from structural data of atomic liquids, like liquid metals. However the effective interaction between micelles is expected to be much softer than atom-atom potentials and to stay finite, even at full overlap, since the pair interactions of the self-assembled soft dumbbells are gaussian-like, with a range $\simeq R_{g}$ and an amplitude $\simeq k_{B} T$. The HNC closure has been shown to be very accurate for such soft, bounded potentials [30], and hence we believe that eq. (12) is sufficient to extract reliable estimates of $v(r)$ without resorting to more advanced inversion schemes which are necessary for strongly repulsive potentials between atoms $[26,27]$.

The accuracy of the HNC inversion procedure is illustrated in Fig. 1, where the pair distribution function $g(r)$ determined in the dumbbell level MC simulations, which is the starting point of our inversion procedure, is compared to the $g(r)$ calculated with the resulting effective micelle-micelle potential $v(r)$ (shown in Fig. 3 ). The agreement is seen to be excellent (a similar conclusive comparison for $f=0.5$ and $\rho / \rho *=5$ was made in ref. [10] and for $f=0.6$ and $\rho / \rho *=3.5$ in 
ref. [11]). It is worth stressing that micelle-level MC simulations are considerably faster (by two orders of magnitude) than the effective dumbbell-level simulations.

A comparison between the $v(r)$ extracted from MC data for $g(r)$ via eqn. (12), based on the two extrapolation procedures described earlier is made in Fig. 2. The agreement is seen to be quite satisfactory, and we will henceforth only present results obtained from MC simulations using the effective potentials derived from the $S(k)$ extrapolation scheme. As expected, the effective potential is seen to be quite soft. Nonetheless there remain large uncertainties for micelle CM-CM distances $r \lesssim$ $2 R_{g}$, because the pair distribution function $g(r)$ is practically zero when micelles overlap strongly, due to a potential barrier of several $k_{B} T$. This is not a major problem for our subsequent results, because strong micelle overlap is a very rare event due to the strong (but finite) repulsion between the coronae.

It is important to stress that the extrapolation and inversion schemes of the pair structure only apply to disordered (fluid) states of the micelle solutions, since they explicitly assume continuous translational invariance, i.e. a homogeneous oneparticle density, which does not hold for ordered (crystalline) states. In the remainder of this paper we will use the micelle-micelle $g(r)$ generated in the MC simulations of references [10] and [11] at the highest $f$-dependent densities where no spontaneous crystallisation of the micelles was observed, to determine the effective pair potential $v(r)$ for three values of $f(f=0.4,0.5$, and 0.6$)$. The corresponding effective pair potentials are plotted in Fig. 3, together with a $v(r)$ at a lower density (for $f=0.4$ ), to illustrate the strong density dependence of $v(r)$. For $r \lesssim 3 R_{g}$, where statistical uncertainties become very large, the potentials are extrapolated linearly to $r=0$, yielding values at full overlap of a few tens of $k_{B} T$. It is these potentials which were used to obtain the data reported in the following sections. We have checked that different extrapolations do not lead to any significant differences in the results.

\section{Micellar crystal structures and Lindemann ratios}

The effective micelle-micelle potentials determined in Section 2 have served as input in extensive MC simulations of samples of several hundred micelles confined to a periodically repeated cubic simulation cell. In order to explore the four crystal structures FCC, BCC, A15, and diamond (2 interpenetrating FCC lattices displaced along the body diagonal of the unit cell by $1 / 4$ of its length), we have placed $N$ effective micelles on the equilibrium lattice sites for each of these crystal structures to construct initial configurations. Thereafter new configurations were generated and accepted or rejected according to the usual Metropolis algorithm [31]. For convenience we use the reduced copolymer density $\rho / \rho *=4 \pi \rho R_{g}^{3} / 3$ as density variable. The corresponding reduced micelle density follows by dividing the reduced copolymer density by the mean aggregation number $n$. For the original system of soft dumbbells the initial configuration is irrelevant to study the fluid phase of self-assembled micelles. However the spontaneous crystallisation of micelles upon increasing density is difficult to trigger and in general provides imperfect structures due to finite size effects. In order to study the properties of crystalline states of different symmetry we have prepared the system in those states as follows. We have placed the system in a periodic external field, acting only on the A blob of the dumbbells, which is attractive and has the symmetry of the required crystalline structure. Starting from a random configuration of dumbbells in the presence of such a field we have observed, during a standard Monte Carlo run, a rapid formation of polydisperse micelles around the lattice sites for all structures considered except the diamond structure. In the latter case the system prefers to occupy only 
the sites of one of the two interpenetrating FCC lattices rather than forming smaller micelles on each lattice site. This is strong indication that the diamond structure is not a stable one for our system. Upon releasing the external field, crystalline arrays of dumbbell micelles in FCC, BCC and A15 structures remain stable if the density is large enough (see discussion below). At lower density the prepared crystals are found to melt rapidly leading the fluid equilibrium state.

Fig. 4a shows the micelle-micelle pair distribution function in the effective micelle representation calculated with an initial FCC configuration (represented by the vertical segments of length equal to the coordination numbers). Clearly the initial crystal has melted and $g(r)$ is typical of a dense fluid. Identical $g(r)$ 's (within the very small statistical uncertainties) are obtained starting from each of the perfect crystal configurations.

Fig. 4b shows the corresponding structure factor $S(k)$. The amplitude of the main peak is close to 2.85 which suggests, according to the Hansen-Verlet criterion [32], that the liquid is very close to freezing. Fig. $4 \mathrm{~b}$ also shows $S(k)$ obtained by a full soft dumbbell simulation. Because the latter is much more computer intensive, the statistical uncertainties are much larger, but the agreement between the structure factor, obtained within the two representations, is excellent, vindicating once more our coarse-graining strategy and the inversion procedure. The good agreement between the two representations is observed throughout the micellar liquid phase. An example of a state $(f=0.4, \rho / \rho *=5)$ close to the cmc is shown in Fig. 5 . Near the cmc, $S(k)$ exhibits a central $(k=0)$ peak associated with the incipient clustering of the dumbbells. It is interesting to note that the coarse-grained effective micelle representation clearly preserves this feature.

Pair distribution functions obtained, starting from the four crystal structures as initial configurations, for $f=0.4$ but at the higher density $\rho / \rho *=7$ are shown in Fig. 6. The peak positions for the FCC, BCC, and A15 crystals are seen to coincide with the corresponding positions of neighbouring shells in a perfect lattice. This is no longer true when the starting configuration is a perfect diamond lattice. The final structure is clearly no longer crystal like, but resembles that of a disordered, glassy system. The diamond structure is thus unstable, and this is confirmed by the time evolution of the mean-square displacement of the micelles from their initial positions, to be discussed later in this Section. Note that a similar instability has been found for the full dumbbell representation as mentioned above.

Very similar behaviour is observed for $f=0.5, \rho / \rho *=6$ and $f=0.6, \rho / \rho *=4$ (not shown). While the FCC, BCC, and A15 structures appear to be stable under those conditions, the diamond lattice always melts into a disordered structure. The reason for this appears to be the low (four-fold) coordination which forces the first shell of neighbours to be at a distance from a central micelle well inside the repulsive part of the effective pair potential $v(r)$, while for the other three crystal structures, the corresponding distances fall into the attractive part of $v(r)$.

The previous observations are confirmed by inspection of the static structure factor $S(k)$, shown in Fig. 7 for $f=0.5, \rho / \rho *=6$ for both the soft dumbbell and the effective micelle representations. The agreement between both sets of data is seen to be reasonable, keeping in mind that the effective micelle representation ignores the polydispersity of micelles observed in the full dumbbell representation.

The $S(k)$ of the FCC, BCC, and A15 crystals are dominated by Bragg peaks at their expected locations, while the structure factor resulting from an initial diamond structure no longer exhibits Bragg peaks, but resembles that of a dense disordered fluid or glass. Similar behaviour is observed for the other two cases studied, $f=0.4, \rho / \rho *=7$ and $f=0.6, \rho / \rho *=4$. Within harmonic theory, the amplitude of the Bragg peaks is governed by the Debye-Waller factor proportional 


\section{Molecular Physics}

8

J.J. Molina et al.

to the mean-square displacement of particle positions $\vec{r}_{i}$ from the equilibrium lattice sites $\vec{r}_{i, 0}$ :

$$
W=\frac{k^{2}}{6}\left\langle\gamma^{2}\right\rangle=\frac{k^{2}}{6}\left\langle\frac{1}{N} \sum_{i=1}^{N}\left(\vec{r}_{i}-\vec{r}_{i, 0}\right)^{2}\right\rangle
$$

namely [33]:

$$
S(k)=1+(N-1) e^{-2 W(k)}
$$

If first-order anharmonic corrections are included, the amplitude of the Bragg peaks is determined by [34]:

$$
S(k)=1+(N-1) \exp \left\{-k^{2} \frac{\left\langle\gamma^{2}\right\rangle}{3}+\frac{\kappa k^{4}}{12}\left(\frac{\left\langle\gamma^{2}\right\rangle}{3}\right)^{2}\right\}
$$

where the non-gaussian parameter $\kappa$ is:

$$
\kappa=3\left(\frac{\left\langle\gamma^{4}\right\rangle}{\left\langle\gamma^{2}\right\rangle^{2}}-1\right)
$$

By fitting the MC $S(k)$ data to the functional forms (14) or (15) we have extracted estimates of the mean-square displacements $\left\langle\gamma^{2}\right\rangle$, which can be compared to the values measured directly in the simulations. The latter estimates, plotted against the number of $\mathrm{MC}$ configurations, are shown in Fig. 8, in the form of the dimensionless Lindemann ratio,

$$
\mathcal{L}=\frac{\sqrt{\left\langle\gamma^{2}\right\rangle}}{d}
$$

where $d$ is the nearest neighbour distance in the crystal lattice at a given micelle density. In all three cases shown, $\mathcal{L}$ is seen to stabilise rapidly at a constant value for the FCC and A15 crystals, while it grows continuously with MC "time" when the initial configuration is the diamond structure, confirming the fact that the latter is unstable i.e. melts into a disordered structure. The BCC structure is somewhat intermediate; for $f=0.5$ and $\rho / \rho *=6$, the corresponding $\mathcal{L}$ stabilises rapidly, but at a value nearly $20 \%$ higher than for FCC and A15. In the case of $f=0.4$, $\rho / \rho *=7, \mathcal{L}_{B C C}$ is more than twice as large as the corresponding FCC and A15 values, and may eventually diverge, suggesting that the BCC phase may only be metastable under those conditions. Finally, for $f=0.6$ and $\rho / \rho *=4, \mathcal{L}_{B C C}$ first appears to stabilise at a value about $20 \%$ above those for FCC and A15, but when the MC run is extended to longer "times", $\mathcal{L}_{B C C}$ is eventually seen to drift continuously to larger values, suggesting that the BCC phase is unstable under those conditions.

To summarise the above results, we have found that in the three cases which we have examined in detail, the diamond structure is always highly unstable while the FCC and A15 phases are always stable. The BCC phase is stable for $f=0.5$, $\rho / \rho *=6$, metastable (and possibly unstable) for $f=0.4, \rho / \rho *=7$, and unstable for $f=0.6, \rho / \rho *=4$. According to the Lindemann melting criterion, a crystal will melt when $\mathcal{L} \simeq 0.15$ (this value is not universal, but depends somewhat on 
the interatomic forces) [16]. We may conclude accordingly that the states $f=0.4$, $\rho / \rho *=7$ and $f=0.5, \rho / \rho *=6$ are well inside the stable FCC and A15 crystal phases, or alternatively that these crystals will melt at somewhat lower micelle densities, while the state $f=0.6, \rho / \rho *=4$ is close to melting of the FCC and A15 crystals.

In Table 1 we compare the Lindemann ratios $\mathcal{L}$ obtained from direct MC estimates to those deduced from an analysis of the structure factors based on eqs. (14) and (15). Good agreement between the two estimates is only achieved when the anharmonic correction (eq. (15)) is taken into account. A reasonable agreement is also observed between the results of the effective micelle and the full dumbbell representations for all structures, confirming the validity of the coarse graining strategy in the symmetry broken phase.

\section{Free Energy Calculations}

In order to assess the relative stabilities of the FCC, A15, and BCC crystal phases, and of the disordered (liquid) micellar phase, we have computed the Helmholtz free energies of all competing phases for the three $(f, \rho / \rho *)$ states considered in the previous Section. Here we limit our study to the effective micelle representation. A free energy calculation for the full dumbbell representation, using a novel method for cluster crystals [35], would be much more demanding and is left for future work.

Since the free energy $F$ at a given state point is not expressible as a Boltzmann weighted statistical average, it cannot be estimated in a single MC simulation. The most direct and robust method is to evaluate $F$ by thermodynamic or Kirkwood coupling constant integration along a reversible path, starting from a reference state point for which the free energy is known [31, 32]. For the free energy of the disordered (fluid) phase, it is natural to take a low density fluid as a reference, for which the free energy can be estimated from the virial expansion [16]. Thermodynamic integration then links the free energies of the low $\left(\rho_{I I}\right)$ and high density $\left(\rho_{I}\right)$ states via:

$$
\frac{\beta F\left(\rho_{I}, T\right)}{N}=\frac{\beta F\left(\rho_{I I}, T\right)}{N}+\int_{\rho_{I I}}^{\rho_{I}} \mathrm{~d} \rho^{\prime} \frac{\beta P\left(\rho^{\prime}, T\right)}{\rho^{\prime 2}}
$$

where $P$ denotes the pressure, which is easily estimated by MC for each intermediate density $\rho^{\prime}$ using the virial theorem $[16,31]$.

However the effective potentials determined in Section 2 exhibit an attractive well of depth $\epsilon$, such that $\epsilon / k_{B} T \gtrsim 1$ (cf. Fig. 3); it is hence to be expected that the thermodynamic path in eq. (18) will cross a vapour-liquid coexistence line, and will hence no longer be reversible. One way to overcome this difficulty is to replace the thermodynamic path (18) along a single isotherm, by two successive paths, one along a higher (supercritical) temperature $T_{I I}$ to go from the low density state $\left(\rho_{I I}, T_{I I}\right)$ to the high density state $\left(\rho_{I}, T_{I I}\right)$, and a second path along the isochore $\rho=\rho_{I}$, to link the high temperature state $\left(\rho_{I}, T_{I I}\right)$ to the final temperature state $\left(\rho_{I}, T_{I}\right)$. Along the latter path the thermodynamic variable to be integrated is the internal energy $U=\partial(\beta F) / \partial \beta$, so that the final expression linking the free 
Molecular Physics

10

J.J. Molina et al.

energies of the reference and actual states is:

$$
\begin{aligned}
\frac{\beta_{I} F\left(\rho_{I}, T_{I}\right)}{N} & =\frac{\beta_{I I} F\left(\rho_{I I}, T_{I I}\right)}{N}+\int_{\rho_{I I}}^{\rho_{I}} \mathrm{~d} \rho^{\prime} \frac{P\left(\rho^{\prime}, T_{I I}\right)}{\rho^{\prime 2}} \\
& +\int_{\beta_{I I}}^{\beta_{I}} \mathrm{~d} \beta^{\prime} \frac{U\left(\rho_{I}, \beta^{\prime}\right)}{N}
\end{aligned}
$$

The free energies of the initial low density, high temperature state is easily evaluated using the second virial coefficient $\left(B_{2}\right)$ approximation for the excess free energy:

$$
\frac{\beta_{I I} F^{e x}\left(\rho_{I I}, T_{I I}\right)}{N}=B_{2}\left(T_{I I}\right) \rho_{I I}
$$

where $B_{2}$ is calculated using the relevant effective pair potential, $\beta_{I I} v(r)=$ $\left(\beta_{I I} / \beta_{I}\right) \beta_{I} v(r)$, with $\beta_{I} v(r)$ the dimensionless effective pair potential appropriate for the final state of interest.

An alternative procedure, which short-cuts the first part of the reversible path (from $\left(\rho_{I I}, T_{I I}\right)$ to $\left(\rho_{I}, T_{I I}\right)$ ) is to use the Widom particle insertion method, which yields the excess part of the chemical potential $\beta_{I I} \mu^{e x}\left(\rho_{I}, T_{I I}\right)$, in a single MC run [31]. The method works well at high temperatures, because of the weakness of the reduced effective potential $\beta_{I I} v(r)$; it would be inadequate at the temperature of interest, when $\beta_{I}>>\beta_{I I}$. The excess Helmholtz free energy $\beta_{I I} F^{e x}\left(\rho_{I}, \beta_{I I}\right) / N$ follows then directly from the thermodynamic relation:

$$
\frac{\beta F^{e x}}{N}=\beta \mu^{e x}-\frac{\beta P^{e x}}{\rho}
$$

To evaluate the free energies of the three stable or metastable crystalline phases (FCC, A15 and BCC), we have used the Frenkel-Ladd method [36], which takes a harmonic Einstein crystal as reference system. The total interaction energy of the Einstein crystal, made up of $N$ independent harmonic oscillators is of the form:

$$
\begin{aligned}
U_{E}\left(\vec{r}^{N}\right) & =E_{M}+U_{H}\left(\vec{r}^{N}\right) \\
& =U\left(\vec{r}_{0}^{N}\right)+\frac{\alpha}{2} \sum_{i=1}^{N}\left(\vec{r}_{i}-\vec{r}_{i, 0}\right)^{2}
\end{aligned}
$$

where the $\vec{r}_{i, 0}$ are the equilibrium lattice positions of the particles, $E_{M}=U\left(\vec{r}_{0}^{N}\right)$ is the Madelung energy (the total energy of a perfect crystal), while $U_{H}\left(\vec{r}^{N}\right)$ is the harmonic energy, with $\alpha$ an appropriate spring constant. If,

$$
U\left(\vec{r}^{N}\right)=\sum_{i<j} v\left(\left|\vec{r}_{i}-\vec{r}_{j}\right|\right)
$$

denotes the total interaction energy of $N$ micelles interacting by the effective pair potential $v(r)$, one introduces a continuous set of systems intermediate between the Einstein crystal and the system of interest, with total potential energy:

$$
U_{\lambda}\left(\vec{r}^{N}\right)=U_{E}\left(\vec{r}^{N}\right)+\lambda\left[U\left(\vec{r}^{N}\right)-U_{E}\left(\vec{r}^{N}\right)\right]
$$

where, by varying the dimensionless coupling constant $\lambda$ from $\lambda=0$ to $\lambda=1$ we 
continuously switch from the reference system to the full system. The free energy of the latter is then given by the following Kirkwood $\lambda$-integration $[16,36]$ :

$$
\begin{aligned}
F & =F_{E}+\int_{0}^{1} \mathrm{~d} \lambda\left\langle U\left(\vec{r}^{N}\right)-E_{M}-U_{H}\left(\vec{r}^{N}\right)\right\rangle_{\lambda} \\
& =F_{E}+\Delta F
\end{aligned}
$$

where the ensemble average $\langle\cdots\rangle_{\lambda}$ is weighted by the Boltzmann factor involving the interaction energy $U_{\lambda}\left(\vec{r}^{N}\right)$, defined in eq. (24). This average is computed by $\mathrm{MC}$ simulations for a discrete number of values of $\lambda$ (typically $4-8$ ). The free energy of the Einstein crystal is known analytically and the integration over $\lambda$ is calculated numerically, using gaussian quadrature.

To improve the numerical accuracy it is convenient to implement the simulations for a fixed $\mathrm{CM}$ of the total system of $N$ micelles, thus preventing the crystal as a whole from drifting when $\lambda \rightarrow 1$, and the harmonic restoring forces deriving from $U_{H}\left(\vec{r}^{N}\right)$ no longer operate [36]. Details of the method, together with the expression for the corresponding constrained Einstein crystal, can be found in reference [31]. The excess free energy per micelle is given by

$\frac{\beta F^{e x}}{N}=\frac{\beta \Delta F^{C M}}{N}+\frac{\beta E_{M}}{N}-\left(1-\frac{1}{N}\right) \ln \left[\rho\left(\frac{2 \pi}{\beta \alpha}\right)^{3 / 2}\right]-\frac{2}{N} \ln N-\frac{1}{N} \ln \sqrt{2 \pi} n_{W S}+1$

where $\Delta F^{C M}$ is the ensemble average defined in (25) evaluated from MC simulations for a fixed CM and $n_{W S}$ is the number of micelles per Wigner-Seitz cell ${ }^{1}$.

The numerical results for the free energies based on the methods described in this Section are presented next.

\section{Competing micellar phases}

We now turn to numerical applications of the free energy expressions of the previous Section, in order to determine the relative stabilities of the disordered and ordered (FCC, A15, BCC) phases. As shown in Section 3, the diamond structure is unstable against melting, at least for the three states $(f ; \rho / \rho *)$ examined in the present work.

Consider first the free energy of the disordered (fluid) phase, which is calculated via eq. (19). The reference state is chosen to be at inverse temperature $\beta_{I I}=$ $\beta_{I} / 15$ and density $\rho_{I I}=\rho_{I} / 100$, and only excess (non-ideal) contributions are considered; the ideal contribution to the free energy is trivially known. Detailed results for the various contributions to $\beta F^{e x} / N$ (eq. (19)) are given below for the state $(0.5 ; 6)$. Using 33 and 14 equally spaced intervals for the integration over density and temperature, respectively, we found the following estimates for the dimensionless integrals:

$$
\begin{gathered}
I_{\rho}=\int_{\rho_{I I}}^{\rho_{I}} \mathrm{~d} \rho^{\prime} \frac{\beta_{I I} P^{e x}\left(\rho^{\prime}, T_{I I}\right)}{\rho^{\prime 2}}=0.1142(1) \\
I_{\beta}=\int_{\beta_{I I}}^{\beta I} \mathrm{~d} \beta^{\prime} \frac{U^{e x}\left(\rho_{I}, \beta^{\prime}\right)}{N}=-12.957(1)
\end{gathered}
$$

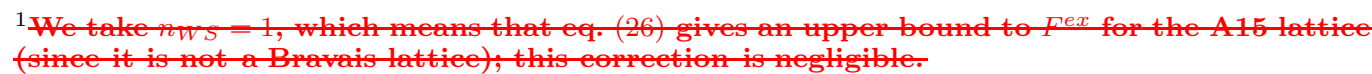


The excess free energy of the reference state is calculated from eq. (20); a simple quadrature yields the second virial coefficient for the state under consideration, with the result $B_{2}=-5.2695 R_{g}^{3}$, so that:

$$
\frac{\beta_{I I} F^{e x}\left(\rho_{I I}, T_{I I}\right)}{N}=-0.0007
$$

gathering results we find the total excess free energy of the liquid phase:

$$
\frac{\beta F_{\text {liq }}^{\text {ex }}}{N}=-12.843(1)
$$

As explained in Section 4, the free energy of the intermediate state $\left(\rho_{I}, \beta_{I I}\right)$ may also be estimated by the Widom insertion method (eq. (21)), which yields:

$$
\frac{\beta_{I I} F^{e x}\left(\rho_{I}, \beta_{I I}\right)}{N}=-0.123(2)
$$

and should be compared to the value $-0.113(0)$ obtained by thermodynamic integration $\left(I_{\rho}+B_{2} \rho_{I}\right)$. The agreement is seen to be satisfactory, and the small discrepancy on this small contribution leads to an error of less than $0.1 \%$ on the total excess free energy.

We have carried out similar calculations for the other $(f, \rho / \rho *)$ states, and the results will be summarised in Table 3 .

We next turn to the free energies of three crystalline phases, which were calculated from eq. (26). Numerical values of various terms are provided in Table 2 for the state $(f=0.5 ; \rho / \rho *=6)$. The total excess free energies of the disordered and ordered phases for all three $(f, \rho / \rho *)$ state points are summarised in Table 3 . Note that the ideal contribution to the free energies, is not included, because $\beta F^{i d} / N$ is identical for all phases at a given density. For all three states, the free energies of the FCC and A15 phases are significantly lower than those of the liquid and of the BCC phase; the latter melts for $f=0.6, \rho / \rho *=4$, and is nearly unstable for $f=0.4, \rho / \rho *=7$. In the latter case, the free energy of the A15 phase lies well below that of the FCC phase, and A15 is hence the stable phase. Note that the A15 phase has the lowest Madelung energy, but the rather large difference in Madelung energies of the FCC and A15 phases is partly cancelled by the larger entropy (the sum of the negative excess term listed in Table 3 and the positive ideal term) of the FCC phase. In the case $f=0.5, \rho / \rho *=6$, the free energy differences are smaller, but A15 still appears to be the most stable phase. In the case $f=0.6, \rho / \rho *=4$, the free energies of the FCC and A15 phases are practically identical and only slightly lower than the corresponding free energy of the liquid phase. Note that in this case the BCC crystal eventually melts (cf. Fig. 8), while the FCC and A15 phases are expected to be close to melting, in view of the value $\mathcal{L} \approx 0.14$ of the Lindemann ratio .

\section{Discussion and Conclusion}

We have investigated the stability of ordered phases of micelles resulting from the self-assembly of a simple model of diblock copolymers in a selective solvent. Our analysis is based on a coarse-grained representation of the micelles, which are treated as monodisperse soft, spherical particles interacting via central forces which derive from an effective, state-dependent potential $v(r)$. The latter is deter- 
mined by an inversion of the pair distribution function of micelles, averaged over copolymer configurations. In order to cope with the relatively small numbers of micelles (typically 50) generated in copolymer-level simulations, we have developed a novel extrapolation/inversion scheme to extract reliable effective potentials from structural MC data in the disordered (fluid) phase. The $v(r)$ turn out to depend sensitively on the copolymer size ratio $f$ and on the overall copolymer density $\rho / \rho *$. This is not surprising, since the mean aggregation number $n$ of the micelles varies significantly upon varying these two basic parameters of the athermal ISS model [11]. The soft effective repulsion between overlapping micelles may be rationalised in terms of the finite repulsive interaction between $A$ and $B$ blobs of the self-assembling copolymers. The physical origin of the attraction between micelles separated by more than their diameter is less obvious, and may be due to a combination of depletion of non-aggregated copolymers, and of exchange of copolymers between neighbouring micelles which leads to fluctuations in their instantaneous aggregation number.

The analysis of the micelle-micelle pair distribution functions, structure factors, mean square displacements, and free energies generated by extensive MC simulations based on the effective pair potentials $v(r)$ for three values of the copolymer size ratio $f$ lead to the following conclusions:

(1) For $f=0.4$ (micelle core smaller than corona), the micelles remain in a disordered fluid state for $\rho / \rho *=6$, but crystalline for $\rho / \rho *=7$. While the diamond structure is unstable (melts), the FCC and A15 structures are stable, while the BCC phase is highly metastable ("overheated"). The A15 phase has a free energy significantly lower than the other phases, and is hence the most stable ordered phase.

(2) For $f=0.5, \rho / \rho *=6, \mathrm{FCC}, \mathrm{A} 15$, and $\mathrm{BCC}$ phases are all stable relative to the disordered (liquid) phase. The excess free energies of the three ordered phases are close (within 1\%), but the A15 structure has again the lowest free energy, and is hence the stable crystal phase.

(3) For $f=0.6$ (micelle core larger than micelle corona), $\rho / \rho *=4$, the BCC phase slowly melts, while the FCC and A15 phases are stable and have nearly identical free energies.

It must be underlined that for all three values of $f$, the A15 structure has the lowest Madelung energy, but this advantage is partially cancelled by the larger entropy of the FCC and BCC phases. The relative stability of the FCC and A15 phases varies with $f$, smaller size ratios $(f<0.6)$ apparently favouring the A15 phase. It would be worthwhile to check this trend in the future, by considering the case $f=0.8$, for which micellar structure data are available [11]. The larger mean aggregation number $(n>100)$ observed for that case points to a "harder" micelle-micelle repulsion, and hence could favour FCC over A15.

The stability of the A15 phase (at least up to $f \simeq 0.6$ ) seems to confirm predictions of Kamien and co-workers for micellization in the solvent-free copolymer melt $[20,21]$. The somewhat unusual A15 structure (which is not a Bravais lattice) may hence be a common phase in self-assembling soft matter systems, although free energy differences with respect to other crystal structures could be very small, as suggested by the present calculation.

Returning finally to the copolymer level, the only limitation of the present coarsegraining approach (which replaces block copolymers by "soft dumbbells") is that the effective interactions between $A$ and $B$ blobs of an $A B$ copolymer were initially determined in the dilute copolymer solution limit [10,11], while clearly local copolymer concentrations within micelles can be quite substantial. This limitation 


\section{Molecular Physics}

can be overcome by switching to a multi-blob representation of each block, similar to a recent proposal for homopolymers in concentrated solutions [17]. Work along these lines is in progress. Recently a novel method has been devised to compute the free energies of cluster crystals of soft core particles[35]. This method could allow the study of the relative stability of crystalline structures of micelles at the full dumbbell representation level and therefore more firmly establish the validity of the coarse grained effective micelle representation.

\section{Acknowledgments}

JJM would like to thank the EACEA for support provided by an Erasmus Mundus Scholarship during his Master project, BC is supported by an EU Marie Curie Fellowship, and CP thanks Schlumberger Cambridge Research and the Royal Society of London for support during visits to Cambridge.

\section{References}

[1]L. Leibler, Macromolecules 13, 1602 (1980).

[2]F.S. Bates, Science 251, 898 (1991).

[3] T.P. Lodge, J. Bang, Z. Li et al., Faraday Discussions 128, 1 (2005).

[4]I.W. Hamley, Block Copolymers in Solution (Wiley, Chichester, 2005).

[5]M.W. Matsen and M. Schick, Phys. Rev. Lett. 72, 2660 (1994).

[6]A. Milchev, A. Bhattacharya and K. Binder, Macromolecules 34, 1881 (2001).

[7]J.A. Anderson, C.D. Lorenz and A. Travesset, J. Chem. Phys 128, 184906 (2008).

[8]S. Woloszczuk, M. Banaszak, P. Knychala and M. Radosz, Macromolecules 41, 5945 (2008).

[9] C.I. Addison, J.P. Hansen, V. Krakoviack and A.A. Louis, Mol. Phys. 103, 3045 (2005).

[10]C. Pierleoni, C. Addison, J.P. Hansen and V. Krakoviack, Phys. Rev. Lett. 96, 128302 (2006).

[11]B. Capone, C. Pierleoni, J.P. Hansen and V. Krakoviack, J. Phys. Chem. B (2008, published on line at http://pubs.acs.org/doi/pdf/10.1021/jp805946z).

[12]P.J. Flory and W.R. Krigbaum, J. Chem. Phys 18, 1086 (1950).

[13] J. Dautenhahn and C.K. Hall, Macromolecules 27, 5399 (1994).

[14]P.G. Bolhuis, A.A. Louis, J.P. Hansen and E.J. Meijer, J. Chem. Phys 114, 4296 (2001).

[15]A.V. Grosberg, P.G. Khalatur and A.R. Khoklov, Mater. Chem. Phys 3, 709 (1982).

[16]J.P. Hansen and I.R. McDonald, Theory of Simple Liquids, 3rd ed. (Academic Press, Amsterdam, 2006).

[17]C. Pierleoni, B. Capone and J.P. Hansen, J. Chem. Phys 127, 171102 (2007).

[18]D. Weaire and R. Phelan, Philos. Magazine Lett. 69, 107 (1994).

[19]P. Ziherl and R.D. Kamien, J. Phys. Chem. B 105, 10147 (2001).

[20]— Phys. Rev. Lett. 85, 3528 (2000).

[21] G.M. Grason, B.A.D. Donna and R.D. Kamien, Phys. Rev. Lett. 91, 058304 (2003).

[22] C.N. Likos, N. Hoffmann, H. Löwen and A.A. Louis, J. Phys. Cond. Matter 14, 7681 (2002).

[23] J.T. Chayes and L. Chayes, J. Stat. Phys 36, 471 (1984).

[24] M.D. Johnson, P. Hutchinson and N.H. March, Proc. R. Soc. London Ser A 282, 283 (1964).

[25] S.K. Mitra, P. Hutchinson and P. Schofield, Philos. Mag. 34, 1087 (1976).

[26] D. Levesque, J.J. Weis and L. Reatto, Phys. Rev. Lett. 54, 451 (1985).

[27]L. Reatto, D. Levesque and J.J. Weis, Phys. Rev. A 33, 3451 (1986).

[28] G. Zerah and J.P. Hansen, J. Chem. Phys 84, 2336 (1986).

[29] L. Verlet, Phys. Rev. 165, 201 (1968).

[30] A. Lang, C.N. Likos, M. Watzlawek and H. Löwen, J. Phys. Cond. Matter 12, 5087 (2000).

[31]D. Frenkel and B. Smit, Understanding Molecular Simulation, 2nd ed. (Academic Press, San Diego, 2002).

[32] J.P. Hansen and L. Verlet, Phys. Rev. 184, 151 (1969).

[33] N.W. Ashcroft and N.D. Mermin, Solid State Physics (Saunders College, Philadelphia, 1976).

[34]E.W. Draeger and D.M. Ceperley, Phys. Rev. B 61, 12094 (2000).

[35] B. Mladek, P. Charbonneau and D. Frenkel, Phys. Rev. Lett. 99, 235702 (2007).

[36]D. Frenkel and A.J.C. Ladd, J. Chem. Phys 81, 3188 (1984). 


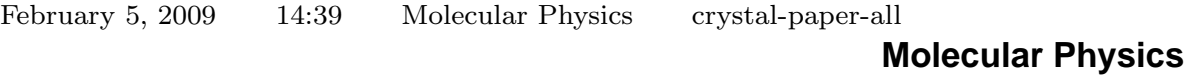

Crystal Stability of Diblock Copolymer Micelles

1

2

3

4

5

6

7

8

9

10

11

12

13

14

15

16

17

18

19

20

21

Table 1. Lindemann ratios estimated by direct MC calculations of $\left\langle\gamma^{2}\right\rangle(\mathcal{L})$, and by fitting eqs. $14\left(\mathcal{L}_{2}\right)$ or $16\left(\mathcal{L}_{4}\right)$ to the structure factor data.

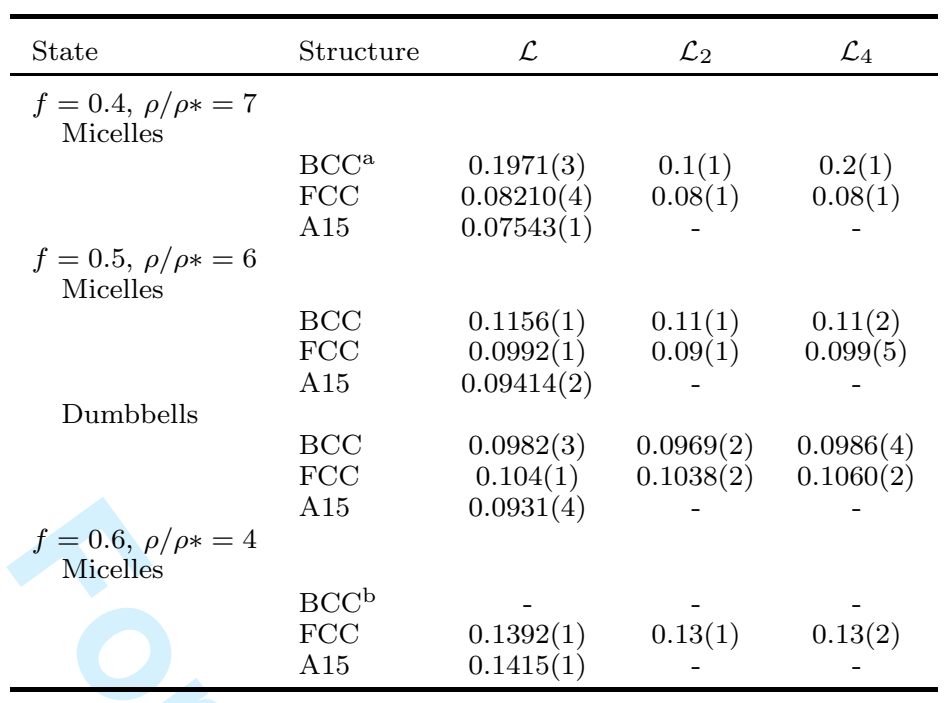

a This system is highly metastable.

b This system is unstable.

Table 2. Excess free energies for FCC, A15 and BCC structures; $f=0.5, \rho / \rho *=6$.

\begin{tabular}{|c|c|c|c|c|c|c|c|}
\hline Lattice & $N$ & $n_{W S}$ & $\beta \alpha$ & $\beta E_{M} / N$ & No. Points & $\beta \Delta F^{C M} / N$ & $\beta F^{e x} / N$ \\
\hline \multirow[t]{4}{*}{$\mathrm{BCC}$} & 432 & 1 & 10.28 & -20.2833 & & & \\
\hline & & & & & 4 & 0.854 & $-13.419(1)$ \\
\hline & & & & & 6 & 0.855 & $-13.418(1)$ \\
\hline & & & & & 8 & 0.852 & $-13.421(1)$ \\
\hline \multirow[t]{4}{*}{$\mathrm{FCC}$} & 500 & 1 & 13.70 & -20.7511 & & & \\
\hline & & & & & 4 & 0.798 & $-13.508(1)$ \\
\hline & & & & & 6 & 0.798 & $-13.508(1)$ \\
\hline & & & & & 8 & 0.798 & $-13.508(1)$ \\
\hline \multirow[t]{4}{*}{ A 15} & 512 & $8^{a}$ & 18.97 & -21.0378 & & & \\
\hline & & & & & 4 & 0.555 & $-13.554(1)$ \\
\hline & & & & & 6 & 0.554 & $-13.555(1)$ \\
\hline & & & & & 8 & 0.5546 & $-13.5546(5)$ \\
\hline
\end{tabular}

a The Wigner-Seitz cell of the A15 lattice is a fourteen-faced polyhedron with 8 sites per cell [19]. 


\section{Molecular Physics}

1

3

4

5

8

9

10

11

12

13

14

15

16

17

18

19

20

21

Table 3. Per particle values for the excess free energy $F^{e x} / N$ (obtained from (26) using a 4 point Gaussian quadrature), internal energy $U^{e x} / N$, entropy $S^{e x} / N=\beta\left(U^{e x}-F^{e x}\right) / N$, and Madelung energy $\beta E_{M} / N$ for the three ordered structures (FCC, BCC, and A15) together with the excess free energy of the disordered (fluid) state.

\begin{tabular}{llccccc}
\hline State & Structure & $N$ & $\beta E_{M} / N$ & $\beta U^{e x} / N$ & $S^{e x} / N$ & $\beta F^{e x} / N$ \\
\hline$f=0.4, \rho / \rho *=7$ & & & & & & \\
& BCC & 432 & -27.2319 & $-26.270(1)$ & $-5.835(1)$ & $-20.4343(4)$ \\
& FCC & 500 & -28.5653 & $-26.9416(3)$ & $-6.0998(3)$ & $-20.8418(1)$ \\
& A15 & 512 & -29.1443 & $-27.5170(3)$ & $-6.4162(3)$ & $-21.1049(1)$ \\
& FLUID & - & - & - & - & - \\
$f=0.5, \rho / \rho *=6$ & & & & & & $-13.419(1)$ \\
& BCC & 432 & -20.2833 & $-18.730(3)$ & $-5.311(3)$ & $-13.508(1)$ \\
& FCC & 500 & -20.7511 & $-19.118(2)$ & $-5.610(2)$ & $-13.554(1)$ \\
& A15 & 512 & -21.0378 & $-19.382(2)$ & $-5.832(2)$ & $-12.843(1)$ \\
& FLUID & 500 & - & $-17.88(1)$ & $-5.03(1)$ & - \\
& & & & & - & $-1.1222(1)$ \\
& BCC & 432 & -7.4076 & & $-1.1215(1)$ \\
& FCC & 500 & -7.4093 & $-5.7663(4)$ & $-4.6441(4)$ & $-1.076(1)$ \\
\hline
\end{tabular}

a The Frenkel-Ladd thermodynamic integration, as applied here, is probably not suitable for this highly metastable BCC phase.

${ }^{\mathrm{b}}$ At this density the system is deeply inside the ordered regime and there is no point to compute the free energy of the fluid phase.

${ }^{\mathrm{c}}$ This structure is unstable. 


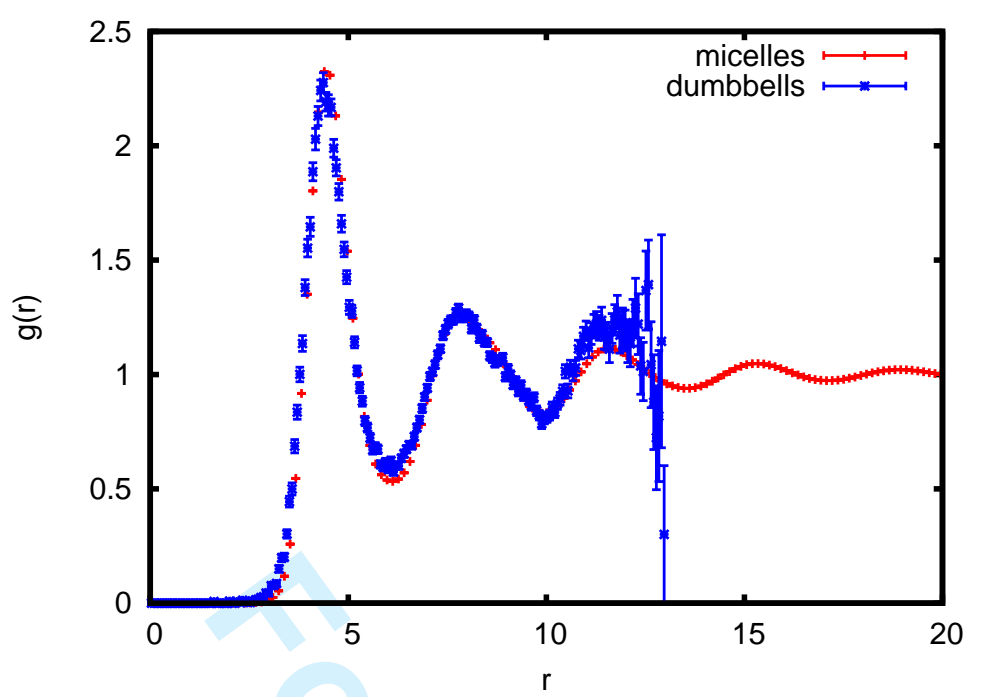

Figure 1. Micelle-micelle pair distribution function $g(r)$ calculated from dumbbell-level and micelle level MC simulations, for $f=0.4$ and $\rho / \rho *=6$. The former data have large statistical uncertainties for the range of micelle-micelle distances $\frac{L}{2}<r<\frac{L \sqrt{3}}{2}$, where the upper limit is the largest distance accessible in a cubic simulation box.

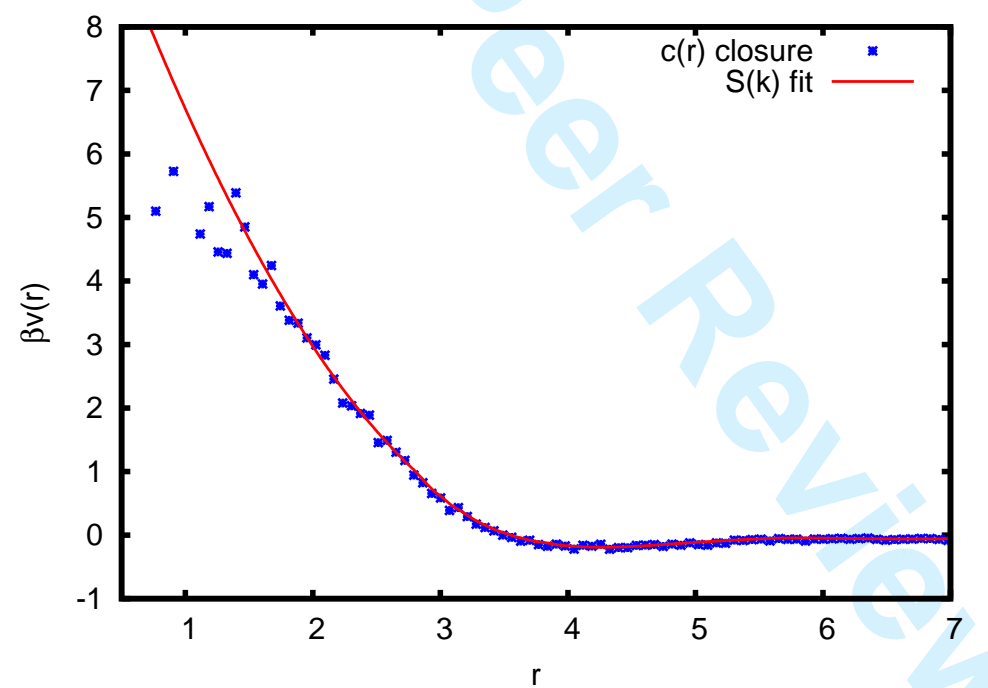

Figure 2. Effective micelle-micelle pair potentials $v(r)$ extracted from data in ref. [11] using the HNC closure with the two extrapolation schemes detailed in Section 2 , for $f=0.4$ and $\rho / \rho *=5$. The solid curve gives the (linearly extrapolated) potential obtained by fitting the structure factor $S(k)$ at small values of $k$ (eqs. (4) and (5)) while the points represent the potential obtained by assuming $c(r)=0$ for $r>r_{c}$ (eq. $(10))$. 


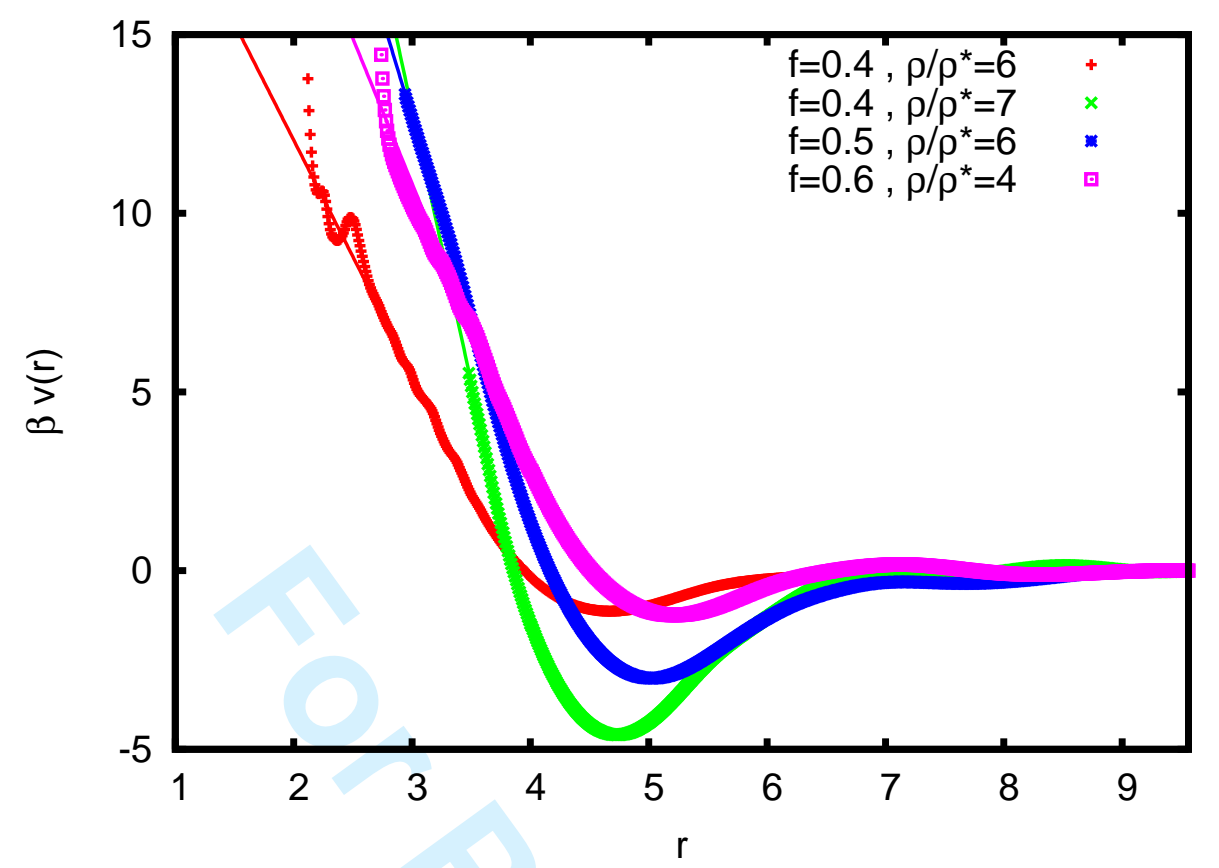

Figure 3. Effective micelle-micelle pair potentials extracted from the HNC closure $(12)$ and the $S(k)$ extrapolation scheme (eqs. (4) and (5)) for 4 couples $(f, \rho / \rho *)$. 

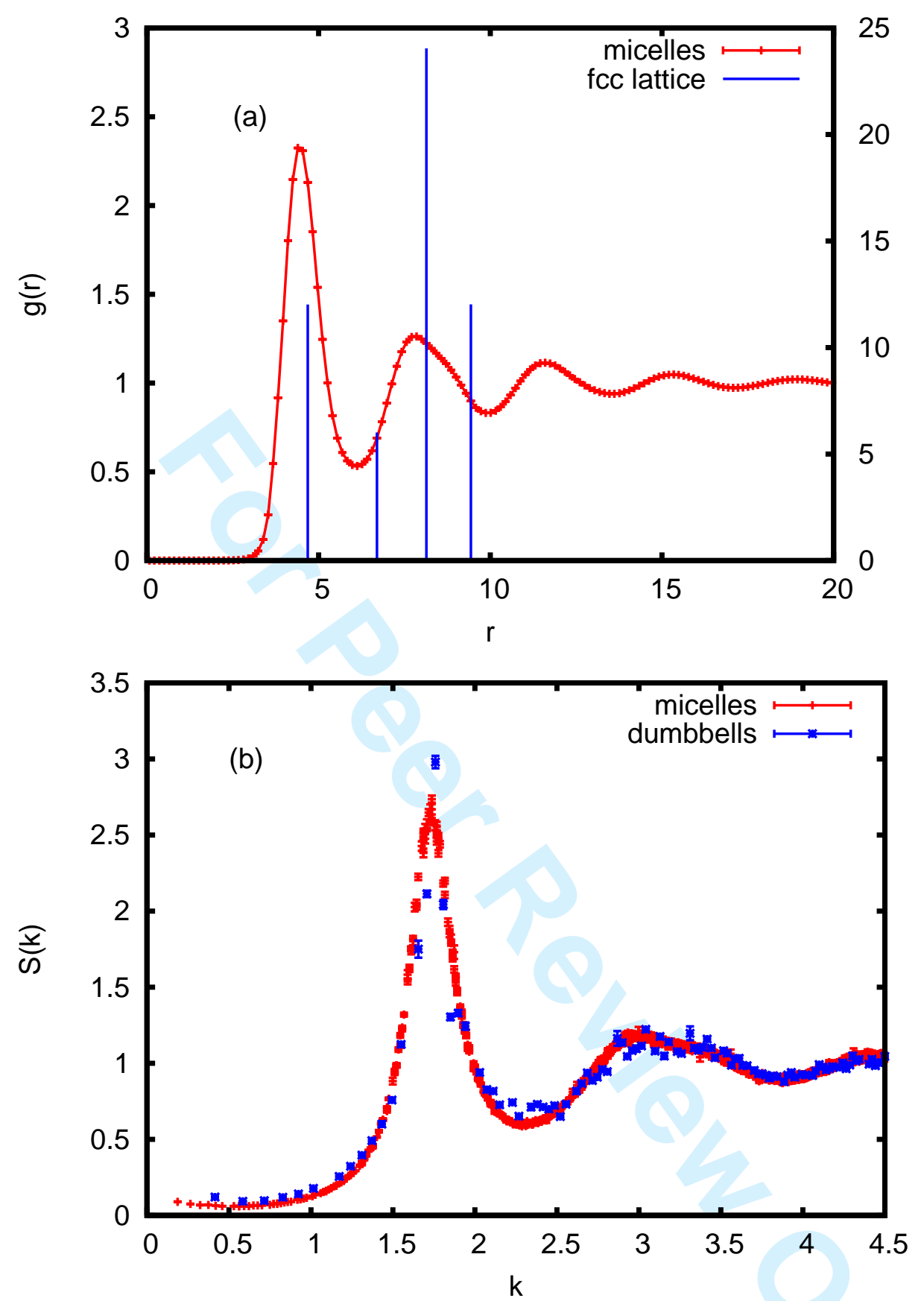

Figure 4. (a) MC-generated $g(r)$ for $f=0.4, \rho / \rho *=6$, starting from a perfect FCC configuration of the micelles. The vertical segments indicate the positions of the first shells of nearest neighbours in the initial FCC lattice, and their height indicates the corresponding coordination number (right hand scale). (b) Corresponding structure factors $S(k)$ (plusses). The structure factor calculated directly from dumbbell level MC simulations is shown as crosses. 


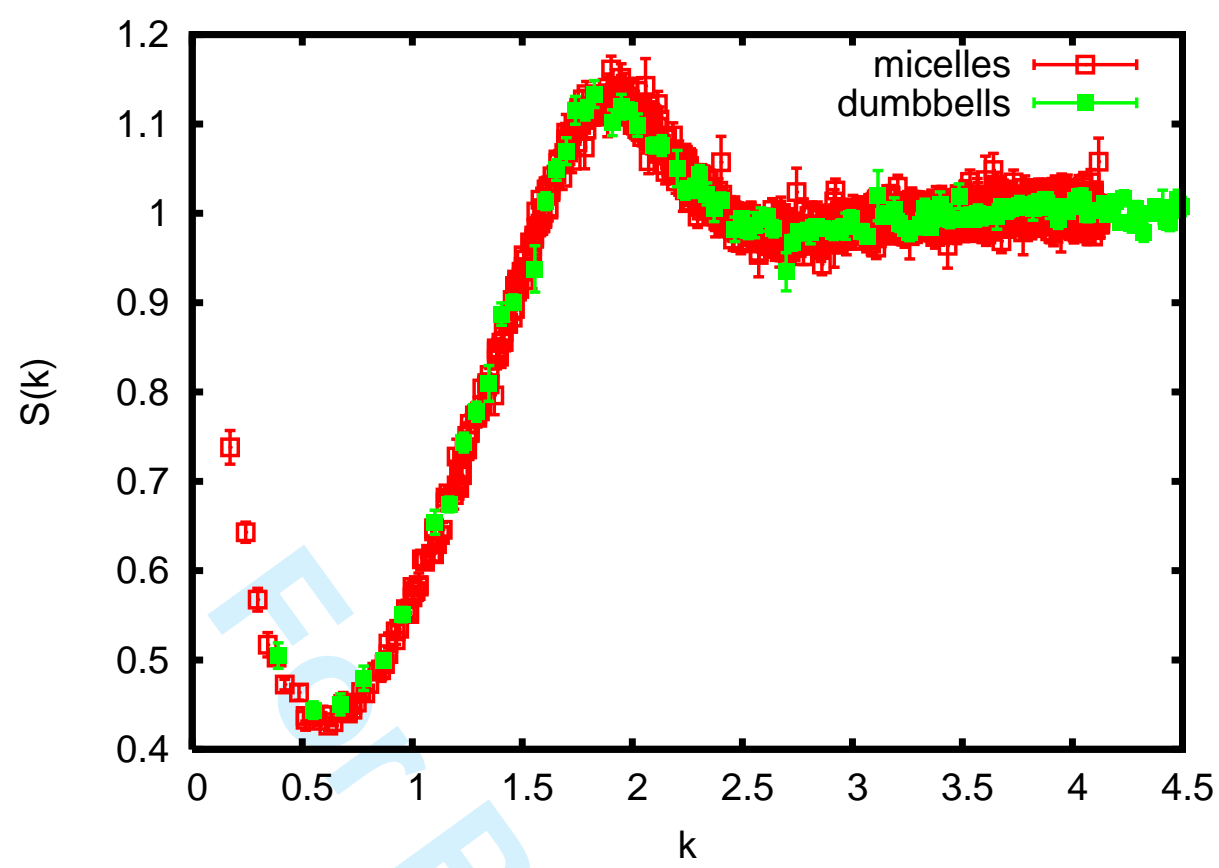

Figure 5. Micelle-micelle structure factors at $f=0.4$ and $\rho / \rho^{*}=5$. Comparison between the full dumbbell representation (solid squares) and the effective micelle model (open squares).
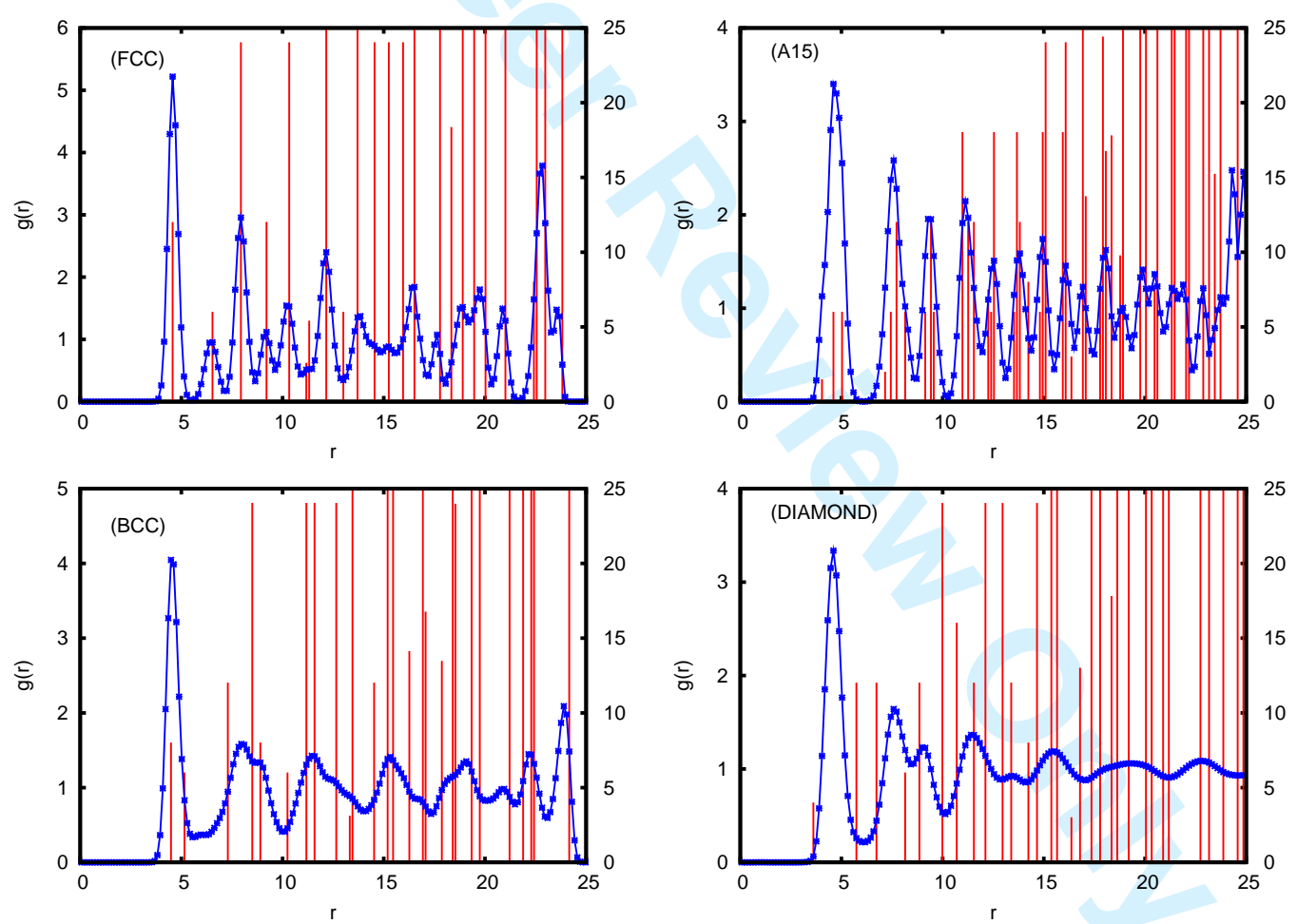

Figure 6. $g(r)$ at $f=0.4, \rho / \rho *=7$, starting from perfect FCC (top left), BCC (bottom left), A15 (top right), and diamond (bottom right) lattice configurations. Vertical segments show positions of neighbouring shells in the corresponding initial perfect lattice configuration. Their heigh indicates the coordination number (right hand scale). 

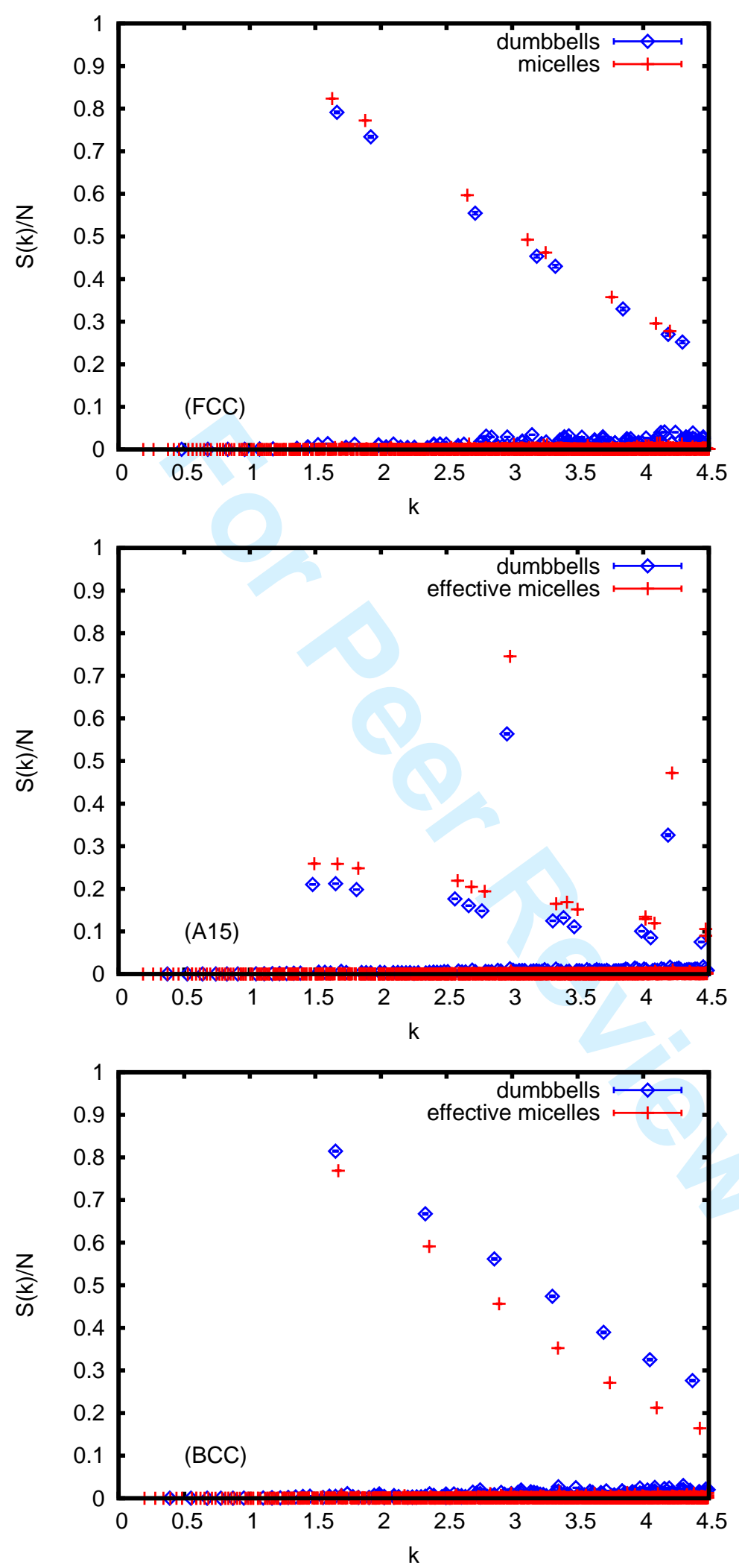

Figure 7. $[S(k) / N]$ vs $k$ at $f=0.5, \rho / \rho *=6$, for FCC, A15, and BCC lattices in both the soft dumbbell (diamonds) and effective micelle (plusses) representations. The Bragg peaks are the large amplitude points decreasing with $k$ according to eq. 14 

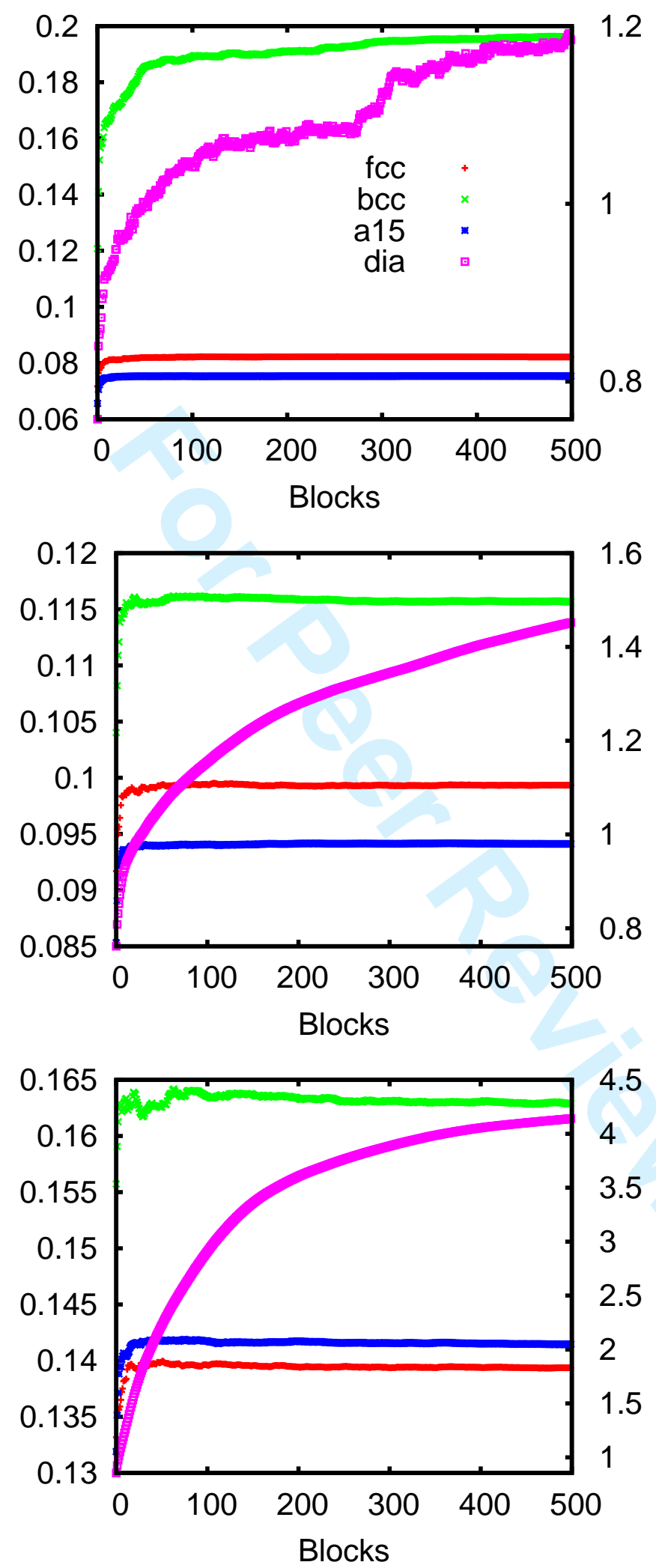

Figure 8. Lindemann ratios (eq. 17) for the three $(f, \rho / \rho *)$ cases considered as functions of the number of MC blocks (each block corresponds to 500 configurations). The FCC,BCC, and A15 values are shown on the left hand axis while the values for the diamond structure are shown on the right hand axis. From top to bottom, $(f=0.4, \rho / \rho *=7),(f=0.5, \rho / \rho *=6)$, and $(f=0.6, \rho / \rho *=4)$. 